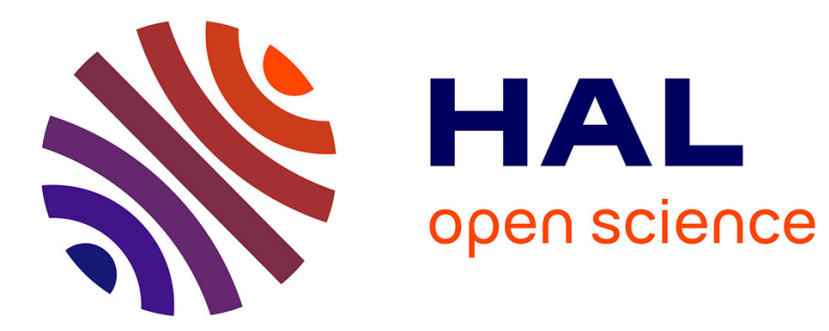

\title{
Un sonar latéral de nouvelle génération
}

\author{
F. Ollivier, P. Alais, N. Cesbron, P. Challande
}

\section{To cite this version:}

F. Ollivier, P. Alais, N. Cesbron, P. Challande. Un sonar latéral de nouvelle génération. Journal de Physique IV Proceedings, 1994, 04 (C5), pp.C5-1309-C5-1312. 10.1051/jp4:19945291 . jpa-00253004

\section{HAL Id: jpa-00253004 https://hal.science/jpa-00253004}

Submitted on 1 Jan 1994

HAL is a multi-disciplinary open access archive for the deposit and dissemination of scientific research documents, whether they are published or not. The documents may come from teaching and research institutions in France or abroad, or from public or private research centers.
L'archive ouverte pluridisciplinaire HAL, est destinée au dépôt et à la diffusion de documents scientifiques de niveau recherche, publiés ou non, émanant des établissements d'enseignement et de recherche français ou étrangers, des laboratoires publics ou privés. 


\title{
Un sonar latéral de nouvelle génération
}

\author{
F. OLLIVIER, P. ALAIS, N. CESBRON et P. CHALLANDE
}

Laboratoire de Mécanique Physique et Expérimentale, Université Pierre et Marie Curie Paris 6, URA 879 du CNRS, 2 place de la Gare de Ceinture, 78210 St-Cyr-l'Ecole, France

\begin{abstract}
Sidescan images of the sea-floor usually suffer from artefacts caused by the attitude variations of the towed platform. In collaboration with IFREMER, we are designing a system that compensates for yaw, pitch and roll in real time, i.e. during the whole ping cycle. The $4 \mathrm{~m}$ long antenna is made of 3 arrays working at $110 \mathrm{kHz}$. It enables ranges up to $1000 \mathrm{~m}$ and the angular resolution is $0.25^{\circ}$ i.e. $1 \mathrm{~m}$ scan resolution for a $250 \mathrm{~m}$ range. The transmit array is made of 36 elements with $80 \mathrm{~mm}$ spacing. There are two identical receiving arrays, containing 32 active pieces each, with $120 \mathrm{~mm}$ spacing. The duplication of the receiving channel will give a bathymetric capability. Data provided by an attitude station are used to control the numerical beam-steering performed at transmit and during receive. On the other hand, 16 focal zones have been set to achieve a constant along-track resolution of $1 \mathrm{~m}$ up to $250 \mathrm{~m}$ range. In addition, focusing reduces the side-lobes level. The experimental results in water tank are in good agreement with the numerical model. First data and images were gathered during a campaign at sea in November 93.
\end{abstract}

\section{Introduction}

Dans le but de réaliser des images du plateau continental, nous développons [1] depuis deux ans en collaboration avec l'IFREMER, un sonar latéral à haute résolution. L'exploration des fonds sous marins au moyen d'engins remorqués est généralement perturbée par les mouvements parasites du véhicule (lacet, tangage, roulis). C'est pourquoi nous avons conçu un système capable en temps réel de compenser l'attitude de son vecteur et d'adapter la focalisation pour optimaliser et homogénéiser la résolution latérale. Par ailleurs un double réseau de réception a été implanté pour recueillir des informations bathymétriques et répondre aux besoins exprimés dans la littérature récente [2][3][4].

La conception des réseaux de transducteurs et la modélisation de leur fonctionnement à constitué une première étape de ce projet. Parallèlement, un système électronique a été entièrement conçu pour le pilotage des réseaux et le traitement des échos. Une première série d'expériences sur maquette dans le bassin du laboratoire a permis de valider le modèle numérique. Cette validation s'est vue confirmée par d'autres expériences sur le système équipé de l'antenne définitive dans le grand bassin de l'IFREMER à Brest. En Novembre 93 enfin, lors d'une première campagne en mer, nous avons pu collecter par faible fond des données bathymétriques ainsi que les premières images. 


\section{Contraintes et conception des antennes}

Le principe du sonar latéral est désormais bien connu et les nombreux systèmes qui l'utilisent varient par leur application, leur performances et leur coût. Notre objectif est la réalisation d'images à haute résolution du plateau continental et l'obtention des profils bathymétriques des zones explorées. L'originalité de ce projet réside dans la capacité du système à compenser les mouvements perturbateurs du véhicule (essentiellement lacet et tangage) dont les antennes sont solidaires.

L'observation latérale du plateau continental, i.e. des fonds compris entre 25 et $300 \mathrm{~m}$, nécessite une portée de l'ordre de $1000 \mathrm{~m}$. La fréquence maximale des signaux acoustiques susceptibles de porter à cette distance aller-retour se situe autour de $100 \mathrm{kHz}$. La fréquence de fonctionnement étant établie, pour obtenir une résolution maximale, on doit disposer d'une antenne linéaire aussi longue que possible. Compte tenu des contraintes liées à la manipulation de l'engin, une dimension longitudinale de $4 \mathrm{~m}$ est raisonnable. Elle permet d'obtenir en champ lointain une résolution angulaire de $0.25^{\circ}$. A une distance de $250 \mathrm{~m}$, on aura ainsi dans le sens de la marche du navire une résolution linéaire de $1 \mathrm{~m}$. Si, pour une telle résolution, le rayonnement doit être aussi étroit que possible dans le plan de l'antenne, il faut en revanche que son ouverture dans le plan vertical soit la plus grande possible afin d'émettre des signaux vers les portées les plus lointaines et recevoir les échos qui en reviennent. La technologie de fabrication des transducteurs en céramiques composites qui a été développée au laboratoire nous a permis de produire des transducteurs de rendement satisfaisant sur une large bande autour de $100 \mathrm{kHz}$. Pour une largeur de $17 \mathrm{~mm}$, ces transducteurs présentent une acceptance angulaire à $-3 \mathrm{~dB}$ de près de $60^{\circ}$ suffisante pour notre application.

Pour réaliser la compensation des mouvements du vecteur, on utilise des antennes à réseaux de transducteurs qui permettent le dépointage des faisceaux par contrôle de la phase des éléments. Une partie du travail numérique de modélisation a consisté à optimiser la géométrie des réseaux pour s'affranchir des artefacts dus au lobes de périodicité. Cette première étude a déterminé le choix de deux réseaux différents pour l'émission et la réception. Leur différence essentielle est dans le pas des éléments jointifs. Il est de $80 \mathrm{~mm}$ pour les émetteurs et de $120 \mathrm{~mm}$ pour les récepteurs. Ce rapport $2 / 3$ entre les pas des 2 réseaux permet, en superposant les diagrammes de rayonnement en transmission et en réception, de réduire de façon suffisante le niveau des lobes de périodicité. D'autre part, le réseau d'émission est plus court de près d'1 $\mathrm{m}$ afin d'avoir un faisceau transmis plus ouvert et assurer une couverture complète de la zone à explorer. Finalement le réseau d'émission comprend 36 transducteurs élémentaires contre 32 pour le réseau de réception. Le dépointage du faisceau à l'émission est possible suivant 16 directions dans un intervalle de $8^{\circ}$. En réception, l'engin adapte la visée selon 32 directions dans le même intervalle et pendant toute la durée de la réception.

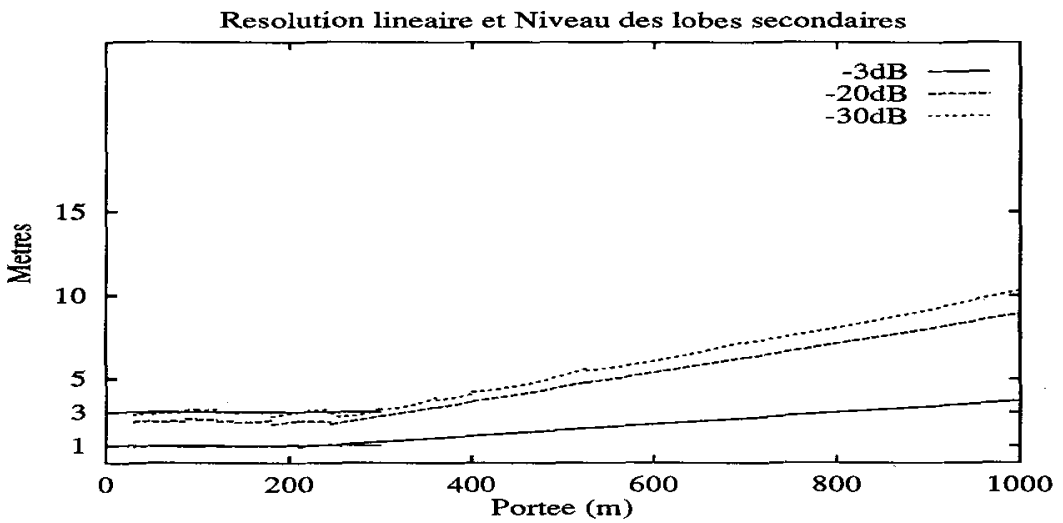

Figure 1

Disposant de réseaux de transducteurs il est aussi possible de réaliser une focalisation dynamique qui permet d'adapter les réseaux à chaque instant de la réception en fonction de la zone dont les échos proviennent. La seconde partie du travail de simulation numérique a cherché à déterminer pour 16 zones focales réparties sur une portée maximale de $1000 \mathrm{~m}$, les meilleurs paramètres de la focalisation. Ces 
paramètres, distance focale théorique et fonction d'apodisation du réseau, sont choisis en sorte d'obtenir jusqu'à une portée de $250 \mathrm{~m}$ une résolution linéaire quasi-constante de $1 \mathrm{~m}$ en conservant des niveaux de lobes secondaires acceptables et ce jusqu'à la portée maximale. Cette bande de résolution constante aura pour effet d'homogénéiser l'image dans la direction transversale à la marche du navire.

La figure 1 représente en fonction de la portée la résolution linéaire théorique du système ainsi que les largeurs à $-20 \mathrm{~dB}$ et $-30 \mathrm{~dB}$ révélatrices du niveau des lobes secondaires.

Pour disposer des informations nécessaires à la réalisation des profils bathymétriques, le réseau de réception est dupliqué. Les deux réseaux de réception sont espacés d'un peu moins de deux longueurs d'onde. Un traitement interférométrique des signaux issus des deux canaux de réception livrera les informations bathymétriques.

\section{Le système électronique et le traitement du signal}

La génération de signaux et le traitement des échos rétro diffusés fait appel à un système électronique dont l'essentiel est numérique.

En émission, on dispose d'une batterie de signaux modulés en amplitude par des fonctions gaussiennes et linéairement en fréquence dans des bandes comprises entre 0 et $3 \mathrm{kHz}$ et centrées sur $112.5 \mathrm{kHz}$. La durée du signal émis peut être choisie entre 1.5 et $8 \mathrm{~ms}$. Des EPROMs servent à stocker l'ensemble. Une autre batterie de mémoires permet le contrôle de la phase de chacun des 36 émetteurs pour le dépointage et l'ouverture du faisceau émis.

En réception, sur chacune des $2 \times 32$ voies programmées selon 32 angles de visée et 16 zones focales, est effectuée une réception hétérodyne corrigée des mouvements du bateau qui translate le signal autour de $4 \mathrm{kHz}$. Les informations déterminant la correction de phase des transducteurs pendant la durée totale d'un cycle sont fournies par une base d'attitude comportant un compas pour le lacet et une base inclinométrique pour le tangage et le roulis. Les signaux accumulés, auxquels est appliqué un gain variable temporellement (T.V.G.), sont échantillonnés à $32 \mu$ s pour chacun des 2 canaux et subissent ensuite une compression d'impulsion par corrélation avec un signal de référence. Cette dernière étape a pour avantage d'augmenter le rapport signal à bruit de façon significative et en proportion du produit BT (bande passante $\mathrm{x}$ durée du signal). Ce même produit détermine la résolution dans la direction transversale à la marche du bateau. Enfin et parallèlement au stockage du signal ainsi traité, une détection d'amplitude échantillonnée à $256 \mu \mathrm{s}$ permet de réaliser et stocker une image brute en temps réel.

\section{Confrontation à l'expérience.}

La partie expérimentale du projet a consisté dans une première étape à tester le système électronique d'une part et à valider le modèle numérique d'autre part. Ceci a été réalisé au laboratoire avec une maquette utilisant une antenne longue de $1 \mathrm{~m}$ dont les réseaux était constitués des éléments de même type que ceux de l'antenne définitive. Les $12 \mathrm{~m}$ de longueur de notre bassin ont permis de tester avec succès le dépointage et la focalisation dynamiques des faisceaux. Les rayonnements expérimentaux de la maquette se sont révélés fidèles à ceux prévus par son modèle numérique.
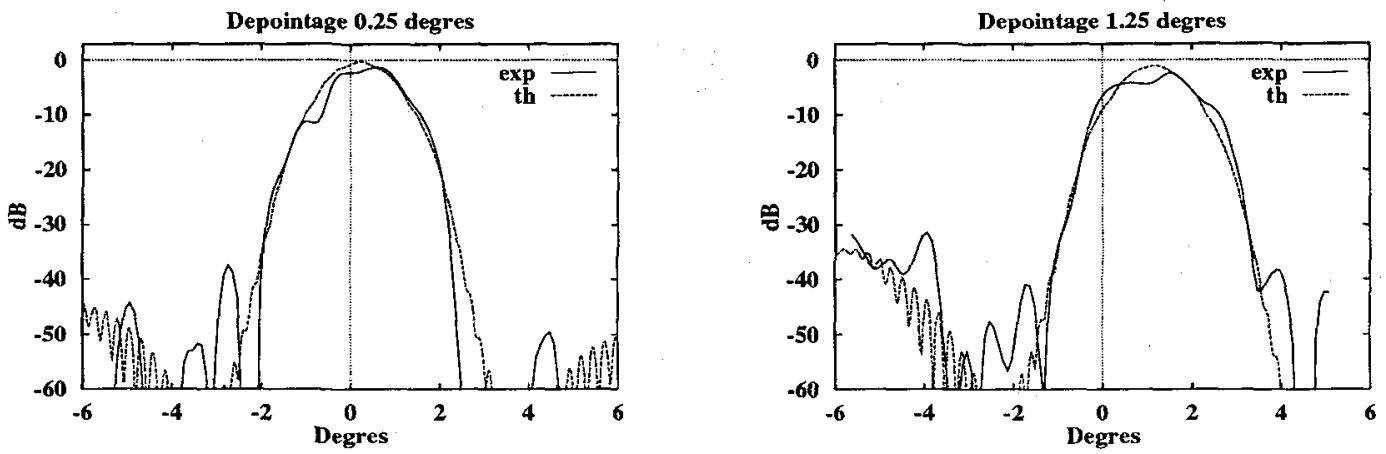

Figure 2 : Rayonnements échographiques de l'antenne de $4 \mathrm{~m}$ - Portée $40 \mathrm{~m}$ 
Dans un second temps, ayant pu disposer du grand bassin de l'IFREMER à Brest, nous avons évalué le rayonnement des différents réseaux de l'antenne de $4 \mathrm{~m}$ de long pour plusieurs dépointages et une portée de $40 \mathrm{~m}$. Le dispositif expérimental utilisait une sonde étalon large bande autour de $100 \mathrm{kHz}$ servant en réception pour la caractérisation du réseau émetteur et en émission pour les réseaux récepteurs. Là encore nous avons constaté le bon accord entre la modélisation et les rayonnements expérimentaux. La figure 2 représente la somme des diagrammes de rayonnement à l'émission et en réception du point de vue du modèle et de l'expérience pour les dépointages à $0.25^{\circ}$ et $1.25^{\circ}$.

La dernière phase expérimentale dans l'état actuel du projet, s'est déroulée à Brest sur le THALIA, navire affrété par l'TFREMER. L'antenne a été fixée sur la coque du navire à environ $1 \mathrm{~m}$ sous la ligne de flottaison. Son inclinaison était de $40^{\circ}$ par rapport à l'horizontale. Les premières images et les données dont on pourra extraire les informations bathymétriques s'y rattachant ont été acquises. Deux de ces images brutes sont présentées ici. Elles n'ont encore subi aucune correction. Chacune des deux images représente une distance à l'antenne de $200 \mathrm{~m}$.

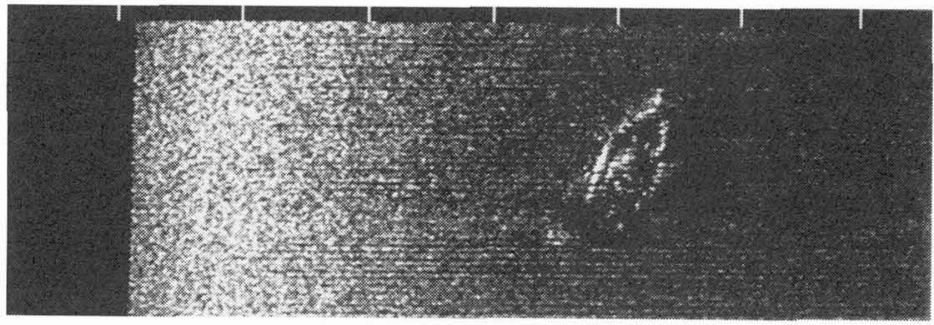

Figure 3 : Image brute de l'épave du Swansea ( $70 \mathrm{~m}$ de long) par $20 \mathrm{~m}$ de fond en rade de Brest

Figure 4 : Image brute de piles d'appontement sur pilotis. Les structures en béton sont révélées par leurs échos spéculaires et leurs ombres portées. On note des détails du terrain et l'écho d'un objet brillant à $175 \mathrm{~m}$ par un fond de $20 \mathrm{~m}$.

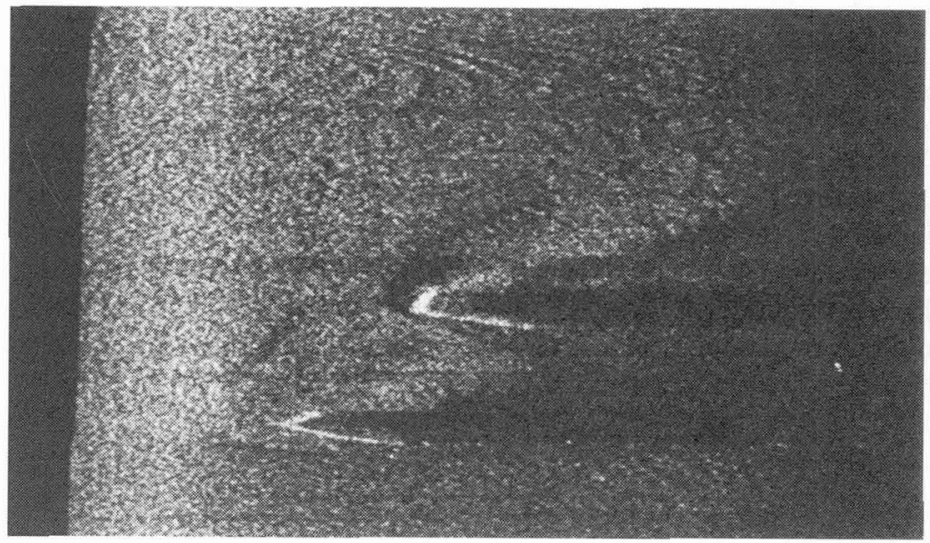

\section{REFERENCES}

[1] P.Alais \& al, "A New Generation Side Scan Sonar", 20éme Congrès International sur l'Imagerie Acoustique à NANKIN, à paraitre dans "Acoustical Imaging 20" (1994).

[2] C. de Moustier, "State of the Art in Swath Bathymetry Survey Systems, Current Practices and New Technology in Ocean Engineering", ASME 29-38 (1988).

[3] B.W.Flemming, \& al., "Recent Developments in Side Scan Sonar Technique", W.G.A. Russel-Cargill Edit.

[4] A.Farcy \& al., "Acoustic Imagery of the Sea Bed", Acoustical Imaging 16, 589-599 (1988) 